\title{
Les relations du virus héréditaire de la Drosophile avec son hôte
}

\author{
par P. L'HERITIER \\ Laboratoire de Génétique, 4, rue Ledru, F 63 - Clermont-Ferrand
}

\begin{abstract}
Résumé
On rencontre couramment dans les populations naturelles de Drosophila melanogaster un Rhabdovirus, appelé $\sigma$, qui, bien qu'artificiellement inoculable, ne se transmet normalement que par les gamètes. Il existe deux catégories de Drosophiles infectés, les nonstabilisées, chez lesquelles le virus n'est présent que dans les tissus somatiques, et les stabilisées, chez lesquelles les cellules germinales également sont infectées. L'état stabilisé est transmis régulièrement par hérédité maternelle et peut prendre naissance à la faveur d'une infection tardive des cystes ovariens chez les femelles non stabilisées. Les modalités de la transmission héréditaire de $\sigma$ sont telles qu'elles permettent au virus de se maintenir dans les populations en dépit de l'action de la sélection naturelle. Cette situation peut servir de modèle pour certaines perspectives dans le domaine de la lutte biologique.
\end{abstract}

\section{Summary}

A Rhabdovirus, called $\sigma$, is quite frequently found in wild populations of Drosophila melanogaster. $\sigma$ is readily transmitted through artificial inoculation, but, in natural conditions, is exclusively propagated by the gametes. There exist two types of $\sigma$-infected flies, the so-called non stabilized, in which the virus occurs in somatic cells only, and the stabilized, in which gonial cells also are infected. The stabilized condition is regularly transmitted through maternal inheritance and may arise by infection of ovarian cysts in non-stabilized females. The hereditary transmission of the virus is efficient enough to allow its preservation in wild populations in spite of natural selection. This biological situation is taken as a model from which some suggestions in the field of biological control of pests are derived. 
La situation biologique du virus héréditaire de la Drosophile (Drosophila melanogaster) est assez singulière. Il s'agit d'une question qui n'a certainement pas de rapport direct avec l'objet de cette réunion. Nul ne songe à lutter contre la Drosophile, qui est un diptère inoffensif, et le virus qu'elle propage, bien qu'étroitement apparenté à des virus pathogènes de vertébrés, parait également tout à fait inoffensif. Ce n'est qu'à titre de modèle que la description de ca cas peut éventuellement intéresser les participants à ce colloque.

Actuellement, le virus héréditaire de la Drosophile est le seul cas d'un microorganisme propagé régulièrement, d'une manière en quelque sorte génétique, par la reproduction de son hôte, à avoir été longuement et minutieusement étudié. Mais, bien entendu, il n'est guère pensable $a$ priori qu'il s'agisse d'une situation biologique unique au monde, et on possède d'ailleurs des indications positives sur l'existence de plusieurs autres cas plus ou moins analogues. Certains d'entre eux, tel cəlui des viroses végétales propagées par les Cicadelles ou les Pucerons, peuvent relever du domaine de la lutte biologique.

On rencontre fréquemment, dans les populations naturelles de Drosophila melanogaster, des individus qui manifestent une réaction physiologique singulière vis-à-vis du gaz carbonique. Alors que ce gaz ne déclenche qu'une narcose facilement réversible chez les mouches normales, les individus qui sont appelés sensibles au $\mathrm{CO}_{2}$ sont définitivement paralysés par un séjour de quelques minutes dans ce gaz. La réaction est très spécifique du gaz carbonique. Son mécanisme physiologique est très mal connu; je ne m'étendrai pas sur cet aspect de la question.

Il se trouve que la sensibilité au $\mathrm{CO}_{2}$ est à peu près l'unique symptôme de la présence dans la Mouche d'un virus connu sous le nom de $\sigma$. Le virion $\sigma$ a été identifié au microscope électronique. Il s'agit d'un virus à enveloppe, qui a la forme générale d'un obus. A l'intérieur de l'obus, on distingue, sur les préparations qui ont subi la coloration négative, la nucléocapside enroulée en spirale. Cette morphologie est caractéristique du groupe des Rhabdovirus, qui comprend un certain nombre d'agents pathogènes de vertébrés, notamment l'agent de la rage, celui de la stomatite vésiculaire et un virus qui ravage les élevages de truites. $\sigma$, bien qu'apparemment tout à fait inoffensif, possède donc de dangereux cousins.

Les rapports de $\sigma$ avec son hôte ont fait l'objet d'une analyse expérimentale approfondie. Je résumerai brièvement les principales conclusions auxquelles elle a conduit.

Dans les conditions biologiques normales, une Drosophile ne peut acquérir le virus par aucune autre voie que la transmission par les gamètes; $\sigma$ n'est donc pas contagieux, mais uniquement héréditaire. Artificiellement, il peut être aisément inoculé.

Un aspect fondamental des relations avec l'hôte est qu'il existe deux catégories de Drosophiles infectées par $\sigma$. Elles ne diffèrent pas par le symptôme physiologique de l'infection, qui est uniformément la sensibilité au $\mathrm{CO}_{2}$, mais par la présence ou l'absence du virus dans les cellules goniales. De ce facteur vont alors dépendre les modalités de la transmission du virus à la descendance. 
Les deux catégories de mouches infectées ont reçu le nom de stabilisées, dans le cas où le virus est présent dans les gonies, et non stabilisées, lorsqu'il est absent.

L'état stabilisé est indéfiniment transmis par hérédité maternelle. Une femelle stabilisée n'engendre donc, en principe, que des descendants stabilisés, quel que soit le mâle qui l'a fécondée. Cette transmission régulière à $100 \%$ admet cependant quelques accidents. Avec une certaine fréquence, le virus se perd dans les cellules germinales, et il apparaît quelques individus sans virus, donc résistants au $\mathrm{CO}_{2}$, dans la descendance des stabilisées. Ces accidents sont qualifiés de décrochages. Avec les bonnes souches de virus, la fréquence des décrochages peut n'être pas plus élevée que celle d'une mutation génique.

Les mâles stabilisés eux aussi transmettent le virus, mais, d'une part, cette transmission est toujours partielle, d'autre part, elle n'aboutit jamais qu'à l'état non stabilisé.

Les Drosophiles non stabilisées sont donc des mouches, dans lesquelles le virus est présent dans les tissus somatiques, mais absent dans les gonies. Chez les mâles, cette situation aboutit à l'absence de toute transmission héréditaire. Lorsqu'il n'est pas déjà inclus dans les spermatogonies, le virus n'atteint jamais les spermatozoïdes. Il en va différemment avec les femelles. Chez celles-ci, avec certaines souches, le virus réussit à passer dans une fraction des œufs, même lorsqu'il est absent dans les gonies. Ce processus d'infection tardive de la lignée germinale dans les femelles non stabilisées a reçu le nom de passage au germen. Ce passage au germen n'atteint qu'une fraction des œufs: une femelle non stabilisée produit donc un mélange d'individus sans virus et d'individus infectés, sensibles au $\mathrm{CO}_{2}$. Parmi ceux-ci, se rencontrent, côte à côte, les deux catégories; les stabilisées et non stabilisées.

Les relations génétiques entre les deux catégories d'individus infectés peuvent donc être résumées de la manière suivante: une stabilisée peut avoir deux origines, être née d'une mère stabilisée (paléo-stabilisée), ou d'un passage au germen (néo-stabilisée). Une non stabilisée ne peut également avoir que deux origines dans les conditions naturelles: avoir reçu le virus d'un père stabilisé, ou provenir d'un passage au germen. L'inoculation artificielle du virus conduit toujours à des non stabilisées, et constitue donc, pour ce type de mouches, une $3^{\circ}$ origine.

L'intérêt de cette situation, du point de vue de la Génétique, est surtout lié à l'existence de l'état stabilisé. Celui-ci correspond, en effet, à une association permanente virus-cellule, association qui est perpétuée par hérédité cellulaire et, au moment de la reproduction sexuée, par hérédité maternelle. Bien qu'avec de notables différences, sur lesquelles je ne veux pas m'étendre, cette situation rappelle celle qui est réalisée dans les bactéries lysogènes.

Un point intéressant dans le présent contexte est l'existence des stabilisées défectives. La variation génétique de $\sigma$ est très étendue et peut affecter tous les aspects de ses relations avec son hôte. Un type de variation particulièrement remarquable aboutit à un état génétique défectif dans lequel le virus devient incapable de subir la maturation. A l'état stabilisé, cette mutation n'est pas létale, car la propagation du virus n'exige, à aucun moment, un processus d'infection des cellules par l'extérieur. 
On observe effectivement des Drosophiles, appelées ultra-p, qui sont des stabilisées pour un virus défectif, incapable de maturation. Ces mouches ne contiennent aucun virus infectieux que l'on puisse extraire par broyat, et ne sont pas sensibles au gaz carbonique. Pour les distinguer des mouches sans virus, il suffit de leur inoculer un extrait de $\sigma$ normal. On constate alors qu'elles manifestent une immunité, d'ailleurs partielle, contre le surinfectant. Les tissus somatiques sont lentement envahis, mais il n'y a jamais aucun passage au germen. L'état ultra-p obéit aux mêmes règles héréditaires que l'état stabilisé, c'est-à-dire qu'il est régulièrement transmis par hérédité maternelle avec quelques décrochages, et n'est jamais transmis par les mâles.

Ayant décrit les aspects essentiels des relations hôte-virus, je voudrais aborder un autre aspect de la situation: l'aspect Génétique des populations. J'ai déjà indiqué que les Drosophiles infectées se rencontraient couramment dans la nature. Aucune enquête très étendue n'a été faite. Les données disponibles paraissent toutefois indiquer que la situation est la suivante. Une certaine proportion d'individus sensibles au $\mathrm{CO}_{2}$ existent un peu partout dans les populations naturelles de Drosophiles. Les prises d'échantillons effectuées n'ont été ni assez nombreuses, ni dispersées sur une aire suffisamment large, pour pouvoir savoir si cette proportion est plus ou moins constante, ou au contraire, varie de place en place dans de larges limites. D'après les observations disponibles, on peut en tout cas considérer la sensibilité au $\mathrm{CO}_{2}$ conférée par $\sigma$ comme une partie intégrante de la variabilité génétique naturelle de Drosophila melanogaster.

Quelques observations fragmentaires paraissent indiquer qu'une situation analogue se retrouve dans d'autres espèces du genre Drosophila.

Cette situation soulève évidemment un problème de dynamique des populations. Tout indique que, dans la nature, $\sigma$ est exclusivement un virus héréditaire : comment se maintient-il dans les populations?

Une première hypothèse est évidemment que, au moins dans certaines circonstances, sa présence confère aux Drosophiles un certain avantage sélectif. L'hypothèse ne peut être exclue. Les expériences de laboratoire ont toutefois apporté des résultats plutôt ambigus sur ce sujet. Quelques données fragmentaires paraissent bien montrer que les Drosophiles infectées sont particulièrement résistantes au froid. Mais, d'un autre côté, certaines souches de $\sigma$ au moins réduisent notablement la ponte des femelles. La variabilité génétique du virus rend, en fait, beaucoup plus difficile qu'il ne paraît au premier abord de déterminer son effet réel sur la Drosophile. Il faudrait travailler avec des souches prises dans la nature, et non pas avec des souches de laboratoire. Or, jusqu'ici, ceci n'a pas été fait.

Un point sur lequel je voudrais insister, car il peut avoir une valeur générale, est que les modalités de la transmission héréditaire de $\sigma$ doivent en fait lui permettre de se maintenir dans les populations naturelles malgré un désavantage sélectif, si celui-ci n'est pas trop fort. Ceci tient au fait que $\sigma$ peut être transmis d'une manière plus efficace qu'un gène.

Sans faire de calculs mathématiques, je me contenterai de montrer qualitativement comment il peut en être ainsi. 
A une certaine génération, supposons que coexistent les trois types d'individus: stabilisés, non stabilisés et sans virus, et que le passage à la génération suivante se fasse par panmixie (fig. 1).

Les décrochages, et éventuellement le désavantage sélectif, tendent à diminuer la fréquence des stabilisées, mais leur pool reçoit un apport venu du passage au germen chez les non stabilisées. Celles-ci devraient diminuer. Elles peuvent ne pas le faire grâce à l'action des ở stabilisés. Tout ovule sans virus fécondé par un spermatozoïde porteur de $\sigma$ devient, en effet, une non stabilisée.

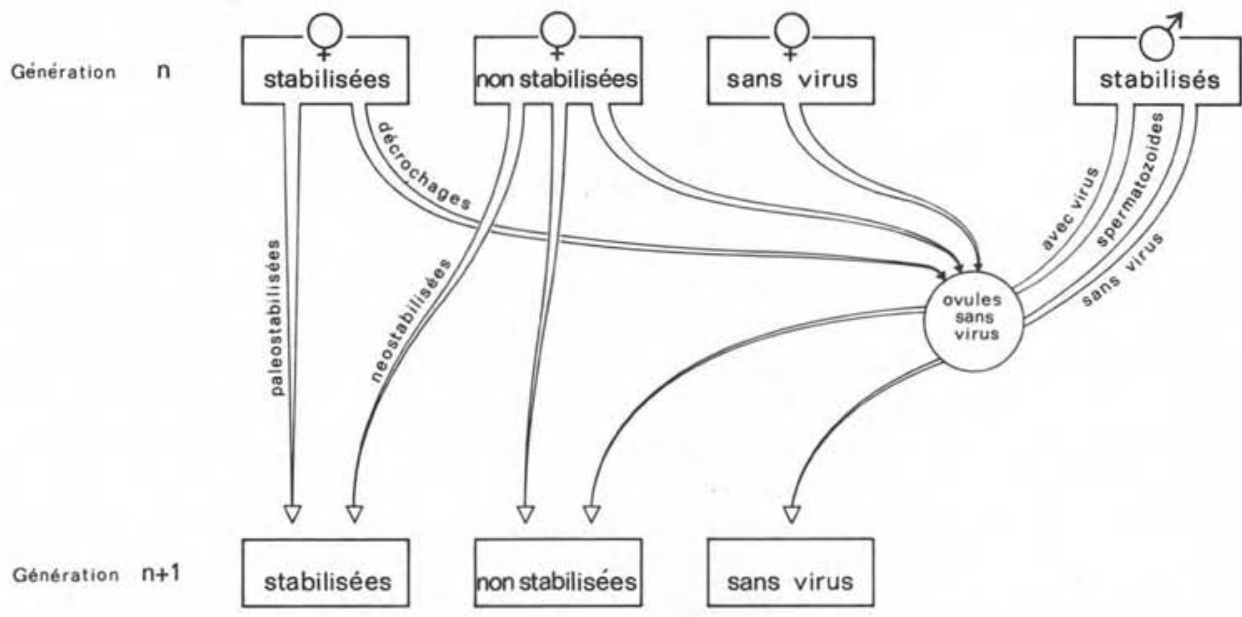

FIG. 1. - Le passage d'une génération à l'autre dans une population de Drosophila melanogaster infectée par $\sigma$

Je pense avoir décrit l'essentiel de la singulière association virus-insecte, que l'on rencontre chez la Drosophile. Au risque de me voir reprocher d'avoir beaucoup d'imagination, je voudrais maintenant faire quelques suggestions qui concernent plus directement le sujet de ce colloque. Imaginant que la situation existant chez la Drosophile se retrouve dans un cas où elle présenterait un danger pour l'homme, ses animaux domestiques ou ses cultures, quels moyens de lutte biologique les connaissances accumulées suggèreraient-elles ?

Il faut, je pense, distinguer les deux cas possibles : celui où c'est le micro-organisme qui est nuisible, l'insecte n'étant qu'un vecteur, et celui d'un micro-organisme inoffensif propagé par un insecte nuisible.

Dans le premier cas, celui du vecteur, il me paraîtrait possible de jouer sur la variabilité génétique du micro-organisme, et de tenter de remplacer, du moins en partie, les formes nuisibles par des formes inoffensives. Le cas des Drosophiles ultra-p, qui propagent un virus défectif et sont immunisées par celui-ci contre un surinfectant non défectif, suggère qu'un tel remplacement pourrait être bénéfique. S'il s'agissait d'un 
vecteur propageant un pathogène de vertébrés, on pourrait même imaginer une véritable vaccination par des insectes porteurs d'une forme non virulente du pathogène. Le fait que $\sigma$ soit très voisin du VSV, pour lequel des diptères piqueurs fonctionnent comme vecteurs, rend cette suggestion moins aventureuse qu'il ne paraît au premier abord.

Dans le deuxième cas, où ce serait l'insecte qui serait nuisible et non plus le microorganisme propagé, on pourrait également tenter de jouer sur la variabilité génétique de celui-ci pour contrôler le porteur. Certaines souches de $\sigma$ réduisent notablement la ponte; leur introduction dans une population naturelle par des lâchers massifs de mâles stabilisés aurait sans doute un effet sur l'effectif des populations.

\section{Bibliographie}

Berkaloff (A.), Bregliano (J.-C.), Ohanessian (A.), 1965. - Mise en évidence de virions dans des Drosophiles infectées par le virus héréditaire. C.R. Ac. Sc., 260, 5956-5959.

Brun (G.), 1963. - Etude de l'association du virus $\sigma$ et de son hôte, la Drosophile : l'état stabilisé. Thèse, Fac. Sc., Orsay.

L'Héritier (Ph.), 1962. — Les relations du virus héréditaire de la Drosophile avec son hôte. Ann. Inst. Pasteur, 102, 511-526.

-, 1969. - Etude des facteurs génétiques contrôlant les relations du virus $\sigma$ et de la Drosophile, son hôte. Ann. Génét., 5, 1-64.

Plus (N.), 1954. - Etude de la multiplication du virus de la sensibilité au $\mathrm{CO}_{2}$ chez la Drosophile. Bull. Biol. Fr. et Belg., 88, 248-293.

Printz (P.), 1969. - Propriétés du virus de la stomatite vésiculaire adapté à la Drosophile. Arc. f. ges. Virusfors., 27, 209-220. 